\title{
Utilization of Dried Rice Distiller's Grains and Solubles (Rice Cake) on Growth Performance of Growing Male Calves
}

\author{
M.C. Pakhira ${ }^{1 *}$, P. Biswas ${ }^{1}$ and M. Mondal ${ }^{2}$ \\ ${ }^{1}$ Department of Livestock Farm Complex, ${ }^{2}$ Department of Veterinary Clinical Complex, F/O- \\ VAS, WBUAFS, Kolkata-700 037, India \\ *Corresponding author
}

\begin{abstract}
A B S T R A C T
\end{abstract}
Thirty two (32) no. of 6 to 9 months of age average body weight $88 \mathrm{~kg}$ were brought from a private dairy farm and were grouped into four $(4 \times 4)$. The treatment groups calf's diet were formulated on iso-caloric and iso- protein basis with the graded level of inclusion

\section{Keywords}

DRDGS $=$ dried rice distillers grains and soluble, ADG = Average daily gain

Article Info

Accepted:

12 August 2018 Available Online:

10 September 2018 dried rice distillers grains and solubles (DRDGS) @ 0\% (TD0), 5\% (TD5), 10\% (TD10), $15 \%$ (TD15) respectively. Both concentrate and roughages were offered as per body weight of calves. Fortnightly feed intake, body weight gain and average daily gain (ADG) were calculated. Feed intake was not significantly $(\mathrm{P}>0.90)$ differ among treatment groups but the group receiving 5\% DRDGS attained highest body weight and the control group attained the least. The average daily gain (ADG) was not affected by dietary supplementation of DRDGS at graded level. The highest ADG was found in TD5 group lowest in control (TD0) group. Overall, the ADG was numerically differ in various experimental groups but statistically not significant $(\mathrm{P}>0.07)$. Again feed intake per unit gain was highest in TD0 group and better was in TD5 group. The TD10 and TD15 group did not show any significant $(\mathrm{P}>0.05)$ result. The dry matter, crude protein, crude fiber, $\mathrm{N}$ free extract, neutral detergent fiber and cellulose digestibility was significantly $(\mathrm{P}<0.05)$ affected by dietary inclusion of graded level of DRDGS in the ration and values were always in higher side but ether extract and hemi-cellulose digestibility was not statistically significant.

\section{Introduction}

In India, livestock rearing is traditional and based on socio-economic considerations (Bhat and Taneja, 1998) due to dearth of enough quality feeds and poor feeding practices. Evidently, feeding alone accounts for about $60-70 \%$ of the total cost of raising the stock. Decreasing area under grass lands, combined with an increasing diversion of crop residues for fuel and industrial uses, is creating an acute shortage of fodder supply for India's livestock. Non-availability of quality fodder in sufficient quantities, in the medium to long term, could negatively affect the health of livestock and, consequently, the production of milk, eggs and meat. However, what has occurred over the past several decades is that the abundance and generally favorable pricing of corn and soybean meal have led to a situation in which other ingredients, which may have been widely studied, have been 
largely overlooked. In the south eastern United States, for example, cottonseed meal, peanut meal and local wheat are all deserving of consideration. Other alternative ingredients which may not have been fully considered in the past are the by-products of the biofuel industry, catfish meal and pearl millet.

Now-a-days microbes act as protein producers. Microbes are used as source of food either directly or as a supplement for pisciculture, livestock and poultry feeding. Of all the microbes, yeast has been commercially exploited most, for the production of alcohol, vitamins and more recently single cell protein (Nikerson and Brawn, 1965). Yeast is very rich in protein and has no toxic substance like other microorganisms like bacteria. Yeast has been used as fodder successfully for certain animals such as horses, cows and as for poultry (Rosales, 1984). Although there is significant interest among ethanol producers to find alternative uses for DDGS, the vast majority of this by-product is sold as a feed ingredient for livestock and poultry feeds. The beverage alcohol industry also produces grain by-products in the form of DDGS or brewer's grains. All of these by-products are nutritionally different and have different economic value in various types of animal $\&$ poultry feeds. In the U. S. finishing beef cattle have successfully been fed as much as $40 \%$ DDGS of ration dry matter as a replacement for corn grain, when corn DDGS to the diet at this level, it is used primarily as an energy source, and supplies more protein and phosphorus than required for finishing feedlot cattle. Feeding DDGS @ 15 to $20 \%$ can increase average daily gain in growing cattle consuming both low quality and high quality forages (Loy et. al., 2003; Morris et. al., 2005), but the reason for increased gain is not fully elucidated. Cattle consuming actively growing forages will respond to undegradable intake protein (UIP supplementation) because the protein in the forage is highly degraded in the rumen (Klopfenstein 1996; Creighton et. al., 2003). Dried distillers grains contain 15 to $20 \%$ UIP (DM basis). However, DDG also contains 8 to $12 \%$ fat (DM basis). Thus, additional energy may also increase ADG. The relative contributions of these nutrients to the performance of cattle grazing forages remains undocumented, and their reported discoveries are important, because DDGS nutrient compositions will change as the milling industry continues to alter the manner in which it process corn (Table 1).

Keeping the view, the rice distillers grains and soluble (DRDGS), a co-product of ethanol industry from rice, was utilized in growing male calves to study its effect on feed intake, growth performance and nutrient utilization.

\section{Materials and Methods}

\section{Animal and experimental design}

Thirty two 6-9 months of age (average body weight) were brought from Ganganagar Dairy Farm, Dum Dum to Department of Animal Nutrition for experimental purpose. Upon arrival male calves were fed concentrate \& roughage along with few hours grazing for fifteen days of adaptation. After adaptation period the animals were grouped into four, one is control and rests three were treatment. Each group having four animals and distribution of animal in each group was made in such a way that the average body weight of all the groups would be similar. All the animals were kept under uniform management condition by housing them in a well-ventilated shed with facilities of individual feeding \& watering. Calves were treated with anthelmentics (Albendazole @ 10mg/kg body weight) before the start of experiment, with 21 days booster recommendation. Thereafter, they are provided at regular interval. They are also treated with external parasites \& fungal infection in any. During the adaptation period 
all the animals were vaccinated against Haemorrhagic Septicaemia, Black Quarter and Foot and Mouth Disease so as to ensure that all the animals were in apparently healthy condition and free from any disease at the onset of experiment. Male calves were fed individually according to their body weight to fulfill the nutrients requirement as per NRC (2001). Measured quantity of treatment diets were offered at 8.00 am everyday. Chapped straw in measured quantity was given twice a day in equal proportion to fill up the bulk of the rumen. Next day in the morning residual feed was collected if any and weighted for calculating the actual feed intake by each animal. Animals had access to adlibitum clean drinking water during the experimental period. Feed intake was adjusted according to mean body weight of each group as well as each animal of the same group every 15 days during the 105 days of experimental period.

\section{Experimental Grouping:}

$\mathrm{TD}_{0}$ - Male calves supplemented with basal diet without DRDGS

$\mathrm{TD}_{5}$ - Male calves supplemented with diet containing 5\% DRDGS

$\mathrm{TD}_{10}$ - Male calves supplemented with diet containing $10 \%$ DRDGS

$\mathrm{TD}_{15}$ - Male calves supplemented with diet containing $15 \%$ DRDGS

\section{Digestibility trial for nutrient utilization}

A digestibility trial of 7 days was carried out during supplemental period for each group of animal to determine the efficiency of nutrient utilization by dried rice cake. During digestibility trial, each animal was offered with measured amounts of total diet (concentrate and straw) and the residues left after 24 hours were collected and weighed.
The faeces voided were collected separately at 24 hours interval for each animal and the amount was quantified. A small amount $(200 \mathrm{~g})$ of faeces was kept daily from each animal for estimation of dry matter, and proximate components (except $\mathrm{CP}$ ). Another small amount $(50 \mathrm{~g})$ of faeces was preserved daily in glass jar containing measured quantity of $20 \%$ (W/V) $\mathrm{H}_{2} \mathrm{SO}_{4}$ for estimation of CP.

\section{Analysis of feeds and faeces}

Estimation of proximate principles viz. CP, $\mathrm{CF}, \mathrm{EE}, \mathrm{NFE}$ and Ash of feeds and faeces were done according to AOAC (1995).

\section{Fiber Fraction}

Fiber fraction viz. NDF, ADF, Cellulose and Hemicelluloses of feeds and faeces were done as per the method Van Soest et al., (1991).

\section{Results and Discussion}

\section{Effect on feed intake}

Fortnightly feed intake (concentrate and roughage) was presented in Table 2 and 3. From the table it has been observed that concentrate and roughage intake by each animal in different experimental groups was not statistically $(\mathrm{P}>0.05) \quad$ significant irrespective of increased level (0 to 15\%) of DRDGS in the ration. Although, the group receiving 5\% DRDGS in the ration, consumes less roughage and more concentrate, but the values were not significant $(\mathrm{P}>0.05)$ statistically.

Again, control group consumed more roughage $(91.36 \mathrm{~kg})$ during the entire experiment but result was not linearly or quadritically significant ( $\mathrm{P}>0.05)$. Precisely, fortnightly or total feed intake (roughage \& concentrate) was not affected by dietary supplementation of DRDGS. 
Larson et al., (1993) reported that feed intake was linearly $(\mathrm{P}<0.01)$ decreased when level of wet distiller's was gradually increased in finishing calf trial.

Ham et al., (1994) reported that when ethanol replaced dry rolled corn at 0,5 or $10 \%$, no linear $(\mathrm{P}>0.10)$ or quadratic effect was detected for dry matter intake in a lamb trial.

Similar observation was also reported by Lodge et al., (1997) who used sorghum and corn distillers grains and soluble in finishing yearling and found no significant difference in dry matter intake per day. Al-Suwaiegh et al., (2002) also reported no difference in dry matter intake in yearling finishing steer using wet corn and sorghum distiller grains and soluble at $35.4 \%$ of DM in the diets. Martin et al., (2007) found no difference in DM intake by beef heifer receiving dried distillers grain.

\section{Effect on body weight and body weight gain}

Fortnightly average body weight (kg), average weight gain $(\mathrm{kg})$ and average daily gain (ADG, in $\mathrm{g}$ ) were presented in Table 4, 5 and 6. Initial average body weight of $\mathrm{TD}_{0}, \mathrm{TD}_{5}$, $\mathrm{TD}_{10}$ and $\mathrm{TD}_{15}$ groups were 89.90, 87.13, 88.20 and $87.50 \mathrm{~kg}$ respectively and final body weight were 127.13, 134.60, 129.67 and $129.10 \mathrm{~kg}$ respectively. From the table it has been noted that $\mathrm{TD}_{5}$ attained the least body weight. Though the body weights were numerically different but statistically $(\mathrm{P}<0.05)$ not significant. Similarly fortnightly average body weight gain in different treatment and control groups were not statistically significant $(\mathrm{P}>0.05)$. Although the DDGS supplemented groups $\left(\mathrm{TD}_{5}, \mathrm{TD}_{10}\right.$ and $\left.\mathrm{TD}_{15}\right)$ gained more body weight but these values were not linearly or quadritically significant $(\mathrm{P}>0.05)$.

Average daily gain (ADG) gm/day, on fortnight basis was not affected by dietary supplementation of DRDGS at graded level. The overall ADG (g/day) on 105 days of growth period were $354.95,452.09,394.95$ and 396.19 respectively for $\mathrm{TD}_{0}, \mathrm{TD}_{5}, \mathrm{TD}_{10}$ and $\mathrm{TD}_{15}$ respectively. The $\mathrm{TD}_{10}$ and $\mathrm{TD}_{15}$ groups showed almost similar result and TD5 showed better result.

The highest $\mathrm{ADG}$ was found in $\mathrm{TD}_{5}$ group \& the lowest was in control (TDO) group. All the above values did not bring forth any significant difference $(\mathrm{P}>0.05)$ among treatment groups as well as with control group.

Daily gain was not affected by increasing graded level of wet distiller's by product as shown by Larson et al., (1993).

Ham et al., (1994) reported no significant (linear or quadratic) difference of ethanol coproduct on lamb growth trial when used at 0,5 or $10 \%$ in the ration and no significant $(\mathrm{P}>0.10)$ affect in daily gains when averaged across protein levels with different distiller's by product. Lodge et al., (1997) also found no difference in daily gain of yearling finishing cattle using corn and sorghum distiller's grains plus soluble. Al-Suwaiegh et al., (2002) reported more daily gain in yearling finishing steers using corn and sorghum distiller's grains and soluble on wet basis $(35.4 \%$ of DM) than control group. Martin et al., (2007) observed no difference in final weight or average daily gain in developing beef heifers using dried distiller's grain.

\section{Effect on feed: gain}

The feed: gain in growing male calves by feeding graded level of dried rice cake was depicted in Table 7. Feed and weight gain were analyzed as a completely randomized design according to the GLM procedure of SPSS (Version 10.0). Animal was used as the experimental unit in all statistical evaluation. 
Table.1 Ingredients \& chemical composition of different experimental rations for growing male calves $(\mathrm{kg} / 100 \mathrm{~kg}$ ration)

\begin{tabular}{|c|c|c|c|c|}
\hline Ingredients & $\mathbf{T D}_{0}$ & $\mathrm{TD}_{5}$ & $\mathbf{T D}_{10}$ & $\mathrm{TD}_{15}$ \\
\hline Maize & 27.0 & 28.4 & 29.3 & 31.3 \\
\hline Soya-DOC & 10.0 & 4.0 & 0.0 & 0.0 \\
\hline MOC & 20.0 & 18.0 & 13.0 & 2.2 \\
\hline RDGS & 0.0 & 5.0 & 10.0 & 15.0 \\
\hline DORB & 40.0 & 41.6 & 44.7 & 48.5 \\
\hline Salt & 1.0 & 1.0 & 1.0 & 1.0 \\
\hline Mineral Mixture & 2.0 & 2.0 & 2.0 & 2.0 \\
\hline Total & 100.0 & 100.0 & 100.0 & $\overline{100.0}$ \\
\hline \multicolumn{5}{|l|}{ Chemical Composition: } \\
\hline Moisture & 9.78 & 9.81 & 9.75 & 9.74 \\
\hline $\mathbf{C P}(\%)$ & 18.83 & 18.84 & 18.84 & 18.85 \\
\hline TDN (\%) & 66.54 & 66.54 & 66.53 & 66.55 \\
\hline $\mathrm{CF}(\%)$ & 9.27 & 9.08 & 8.79 & 8.37 \\
\hline EE $(\%)$ & 2.73 & 2.86 & 2.81 & 2.48 \\
\hline NFE (\%) & 49.48 & 49.31 & 49.58 & 50.2 \\
\hline ADF (\%) & 16.58 & 16.21 & 15.56 & 14.43 \\
\hline NDF (\%) & 28.23 & 27.97 & 27.31 & 26.06 \\
\hline Hemi cellulose $(\%)$ & 11.65 & 11.76 & 11.75 & 11.63 \\
\hline Cellulose (\%) & 14.21 & 13.18 & 12.08 & 10.92 \\
\hline $\operatorname{Ash}(\%)$ & 9.91 & 10.10 & 10.23 & 10.36 \\
\hline DM (\%) & 90.22 & 90.11 & 90.25 & 90.26 \\
\hline
\end{tabular}


Table.2 Fortnightly average concentrate intake $(\mathrm{kg}) /$ animal by feeding of sun dried rice cake at graded level (DRDGS) in growing male calves

\begin{tabular}{|c|c|c|c|c|c|c|c|}
\hline \multirow[t]{2}{*}{ Day } & \multirow[t]{2}{*}{$\mathrm{TD}_{0}$} & \multirow[t]{2}{*}{$\mathrm{TD}_{5}$} & \multirow[t]{2}{*}{$\mathrm{TD}_{10}$} & \multirow[t]{2}{*}{$\mathrm{TD}_{15}$} & \multirow[t]{2}{*}{$\begin{array}{c}\text { Pooled } \\
\text { SE }\end{array}$} & \multicolumn{2}{|c|}{$\begin{array}{c}\text { Significant Effect of } \\
\text { DRDGS }\end{array}$} \\
\hline & & & & & & Linear & Quadratic \\
\hline $0-15$ & 28.06 & 27.20 & 27.55 & 27.29 & 4.05 & 0.917 & 0.943 \\
\hline $16-30$ & 29.40 & 29.01 & 29.22 & 29.18 & 4.23 & 0.982 & 0.968 \\
\hline $31-45$ & 31.26 & 31.37 & 31.15 & 31.11 & 4.37 & 0.974 & 0.986 \\
\hline $46-60$ & 32.97 & 33.24 & 32.81 & 32.65 & 4.57 & 0.948 & 0.964 \\
\hline $61-75$ & 34.70 & 35.93 & 34.63 & 34.27 & 4.75 & 0.905 & 0.871 \\
\hline $76-90$ & 36.29 & 37.95 & 36.35 & 36.07 & 4.95 & 0.921 & 0.850 \\
\hline $91-105$ & 38.03 & 39.83 & 38.37 & 38.38 & 5.04 & 0.987 & 0.863 \\
\hline
\end{tabular}

Table.3 Fortnightly average roughage intake $(\mathrm{kg}) /$ animal by feeding of sun dried rice cake at graded level (DRDGS) in growing male calves

\begin{tabular}{|c|c|c|c|c|c|c|c|}
\hline \multirow[t]{2}{*}{ Day } & \multirow[t]{2}{*}{$\mathrm{TD}_{0}$} & \multirow[t]{2}{*}{$\mathrm{TD}_{5}$} & \multirow[t]{2}{*}{$\mathbf{T D}_{10}$} & \multirow[t]{2}{*}{$\mathrm{TD}_{15}$} & \multirow[t]{2}{*}{$\begin{array}{c}\text { Pooled } \\
\text { SE }\end{array}$} & \multicolumn{2}{|c|}{$\begin{array}{c}\text { Significant Effect of } \\
\text { DRDGS }\end{array}$} \\
\hline & & & & & & Linear & Quadratic \\
\hline $0-15$ & 10.93 & 10.36 & 10.81 & 10.89 & 1.58 & 0.964 & 0.842 \\
\hline $16-30$ & 11.90 & 10.76 & 11.47 & 11.57 & 1.59 & 0.971 & 0.707 \\
\hline $31-45$ & 12.45 & 12.02 & 12.18 & 12.30 & 1.59 & 0.970 & 0.868 \\
\hline $46-60$ & 13.09 & 13.30 & 12.77 & 12.85 & 1.71 & 0.873 & 0.971 \\
\hline $61-75$ & 13.72 & 13.71 & 13.47 & 13.51 & 1.73 & 0.914 & 0.988 \\
\hline $76-90$ & 14.32 & 14.59 & 14.30 & 14.06 & 1.79 & 0.897 & 0.890 \\
\hline $91-105$ & 14.95 & 15.13 & 14.93 & 14.94 & 1.84 & 0.982 & 0.960 \\
\hline
\end{tabular}

Table.4 Fortnightly body weight $(\mathrm{kg})$ by feeding of sun dried rice cake at graded level (DRDGS) in growing male calves

\begin{tabular}{|c|c|c|c|c|c|c|c|}
\hline \multirow[t]{2}{*}{ Day } & \multirow[t]{2}{*}{$\mathrm{TD}_{0}$} & \multirow[t]{2}{*}{$\mathrm{TD}_{5}$} & \multirow[t]{2}{*}{$\mathrm{TD}_{10}$} & \multirow[t]{2}{*}{$\mathrm{TD}_{15}$} & \multirow[t]{2}{*}{$\begin{array}{l}\text { Pooled } \\
\text { SE }\end{array}$} & \multicolumn{2}{|c|}{$\begin{array}{c}\text { Significant Effect of } \\
\text { DRDGS }\end{array}$} \\
\hline & & & & & & Linear & Quadratic \\
\hline 0 & 89.90 & 87.13 & 88.20 & 87.50 & 13.05 & 0.919 & 0.939 \\
\hline 15 & 94.17 & 92.93 & 93.60 & 93.57 & 13.58 & 0.986 & 0.966 \\
\hline 30 & 100.07 & 100.60 & 99.80 & 99.77 & 13.99 & 0.979 & 0.984 \\
\hline 45 & 105.60 & 107.53 & 105.00 & 104.67 & 14.65 & 0.937 & 0.940 \\
\hline 60 & 111.10 & 115.13 & 111.00 & 109.97 & 15.21 & 0.915 & 0.872 \\
\hline 75 & 116.27 & 121.70 & 116.50 & 115.70 & 15.82 & 0.925 & 0.847 \\
\hline 90 & 121.43 & 127.27 & 123.00 & 123.03 & 16.26 & 0.935 & 0.807 \\
\hline 105 & 127.13 & 134.60 & 129.67 & 129.10 & 16.57 & 0.990 & 0.815 \\
\hline
\end{tabular}


Table.5 Fortnightly body weight gain $(\mathrm{kg})$ by feeding of sun dried rice cake at graded level (DRDGS) in growing male calves

\begin{tabular}{|c|c|c|c|c|c|c|c|}
\hline \multirow[t]{2}{*}{ Day } & \multirow[t]{2}{*}{$\mathrm{TD}_{0}$} & \multirow[t]{2}{*}{$\mathrm{TD}_{5}$} & \multirow[t]{2}{*}{$\mathrm{TD}_{10}$} & \multirow[t]{2}{*}{$\mathrm{TD}_{15}$} & \multirow[t]{2}{*}{$\begin{array}{l}\text { Pooled } \\
\text { SE }\end{array}$} & \multicolumn{2}{|c|}{$\begin{array}{c}\text { Significant Effect of } \\
\text { DRDGS }\end{array}$} \\
\hline & & & & & & Linear & Quadratic \\
\hline $0-15$ & 4.27 & 5.80 & 5.40 & 6.07 & 0.817 & 0.208 & 0.610 \\
\hline $16-30$ & 5.90 & 7.67 & 6.20 & 6.20 & 0.626 & 0.845 & 0.196 \\
\hline $31-45$ & 5.53 & 6.93 & 5.20 & 4.90 & 0.888 & 0.339 & 0.403 \\
\hline $46-60$ & 5.50 & 7.60 & 6.00 & 5.30 & 0.900 & 0.600 & 0.159 \\
\hline $61-75$ & 5.17 & 6.57 & 5.57 & 5.73 & 0.733 & 0.840 & 0.448 \\
\hline $76-90$ & 5.16 & 5.97 & 6.43 & 7.33 & 0.638 & 0.070 & 0.743 \\
\hline 91-105 & 5.70 & 6.93 & 6.67 & 6.07 & 0.696 & 0.572 & 0.158 \\
\hline
\end{tabular}

Table.6 Fortnightly average daily gain (g) by feeding of sun dried rice cake at graded level (DRDGS) in growing male calves

\begin{tabular}{|c|c|c|c|c|c|c|c|}
\hline \multirow[t]{2}{*}{ Day } & \multirow[t]{2}{*}{$\mathrm{TD}_{0}$} & \multirow[t]{2}{*}{$\mathrm{TD}_{5}$} & \multirow[t]{2}{*}{$\mathrm{TD}_{10}$} & \multirow[t]{2}{*}{$\mathrm{TD}_{15}$} & \multirow[t]{2}{*}{$\begin{array}{c}\text { Pooled } \\
\text { SE }\end{array}$} & \multicolumn{2}{|c|}{$\begin{array}{c}\text { Significant Effect of } \\
\text { DRDGS }\end{array}$} \\
\hline & & & & & & Linear & Quadratic \\
\hline $0-15$ & 284.44 & 386.67 & 360.00 & 404.44 & 54.46 & 0.208 & 0.610 \\
\hline $16-30$ & 393.33 & 511.11 & 413.33 & 413.33 & 41.74 & 0.845 & 0.196 \\
\hline $31-45$ & 377.78 & 462.22 & 346.67 & 326.67 & 59.18 & 0.339 & 0.403 \\
\hline $46-60$ & 366.67 & 506.67 & 400.00 & 353.33 & 60.03 & 0.600 & 0.159 \\
\hline $61-75$ & 344.44 & 437.78 & 371.11 & 382.22 & 51.51 & 0.845 & 0.448 \\
\hline $76-90$ & 366.67 & 397.78 & 428.89 & 488.89 & 42.51 & 0.070 & 0.743 \\
\hline 91-105 & 357.78 & 462.22 & 444.44 & 404.44 & 46.40 & 0.572 & 0.158 \\
\hline
\end{tabular}

Table.7 Fortnightly feed: gain ratio by feeding of sun dried rice cake at graded level (DRDGS) in growing male calves

\begin{tabular}{|c|c|c|c|c|c|c|c|}
\hline \multirow[t]{2}{*}{ Day } & \multirow[t]{2}{*}{$\mathrm{TD}_{0}$} & \multirow[t]{2}{*}{$\mathrm{TD}_{5}$} & \multirow[t]{2}{*}{$\mathrm{TD}_{10}$} & \multirow[t]{2}{*}{$\mathrm{TD}_{15}$} & \multirow[t]{2}{*}{$\begin{array}{c}\text { Pooled } \\
\text { SE }\end{array}$} & \multicolumn{2}{|c|}{$\begin{array}{c}\text { Significant Effect of } \\
\text { DRDGS }\end{array}$} \\
\hline & & & & & & Linear & Quadratic \\
\hline $0-15$ & 9.29 & 6.37 & 7.08 & 6.67 & 0.91 & 0.116 & 0.202 \\
\hline $16-30$ & 7.07 & 5.08 & 6.54 & 6.70 & 0.72 & 0.915 & 0.174 \\
\hline $31-45$ & 7.84 & 6.33 & 8.35 & 9.01 & 0.88 & 0.197 & 0.255 \\
\hline $46-60$ & 8.98 & 6.07 & 7.51 & 8.76 & 1.08 & 0.878 & 0.092 \\
\hline $61-75$ & 9.62 & 7.58 & 8.65 & 8.28 & 0.78 & 0.424 & 0.314 \\
\hline $76-90$ & 9.84 & 8.65 & 7.88 & 6.79 & 1.18 & 0.097 & 0.968 \\
\hline $91-105$ & 10.13 & 7.84 & 9.07 & 9.03 & 1.22 & 0.715 & 0.385 \\
\hline
\end{tabular}


Table.8 Effect on nutrient utilization by feeding graded level of dried Rice cake in growing calves

\begin{tabular}{|c|c|c|c|c|c|c|c|}
\hline \multirow[t]{2}{*}{ Particulars } & \multirow[t]{2}{*}{ CD0 } & \multirow[t]{2}{*}{ TD5 } & \multirow[t]{2}{*}{ TD10 } & \multirow[t]{2}{*}{ TD15 } & \multirow[t]{2}{*}{$\begin{array}{c}\text { Pooled } \\
\text { SE }\end{array}$} & \multicolumn{2}{|c|}{$\begin{array}{c}\text { Significant Effect of } \\
\text { DRDGS }\end{array}$} \\
\hline & & & & & & Linear & Quadratic \\
\hline \multicolumn{8}{|l|}{ Dry matter } \\
\hline Intake (kg) & 3.319 & 3.319 & 3.234 & 3.248 & 0.434 & 0.892 & 0.461 \\
\hline Outgo (kg) & 1.372 & 1.372 & 1.301 & 1.284 & 0.162 & 0.796 & 0.848 \\
\hline Digestibility (\%) & 58.02 & 58.55 & 59.64 & 60.49 & 0.589 & 0.012 & 0.797 \\
\hline \multicolumn{8}{|l|}{ Crude Protein } \\
\hline Intake (g) & 499.84 & 527.73 & 512.13 & 514.89 & 71.72 & 0.887 & 0.835 \\
\hline Outgo (g) & 184.36 & 186.22 & 177.72 & 173.52 & 24.49 & 0.718 & 0.904 \\
\hline Digestibility (\%) & 63.12 & 65.02 & 65.15 & 66.38 & 0.607 & 0.007 & 0.594 \\
\hline \multicolumn{8}{|l|}{ Crude Fiber } \\
\hline Intake (g) & 525.85 & 545.39 & 525.94 & 516.37 & 67.88 & 0.931 & 0.815 \\
\hline Outgo (g) & 213.85 & 207.61 & 190.89 & 189.87 & 25.13 & 0.453 & 0.920 \\
\hline Digestibility (\%) & 59.33 & 61.94 & 63.77 & 63.36 & 0.579 & 0.001 & 0.031 \\
\hline \multicolumn{8}{|l|}{ Ether Extract } \\
\hline Intake (g) & 96.44 & 104.80 & 100.77 & 92.71 & 13.47 & 0.869 & 0.555 \\
\hline Outgo (g) & 30.11 & 30.06 & 29.14 & 26.77 & 3.89 & 0.547 & 0.773 \\
\hline Digestibility (\%) & 68.77 & 71.52 & 71.11 & 71.21 & 0.789 & 0.086 & 0.132 \\
\hline \multicolumn{8}{|c|}{ Nitrogen Free Extract } \\
\hline Intake (g) & 1700.51 & 1783.76 & 1743.49 & 1765.96 & 234.33 & 0.869 & 0.871 \\
\hline Outgo (g) & 627.62 & 640.48 & 598.54 & 593.95 & 75.32 & 0.547 & 0.911 \\
\hline Digestibility (\%) & 63.08 & 64.14 & 65.55 & 66.26 & 0.507 & 0.086 & 0.739 \\
\hline \multicolumn{8}{|c|}{ Neutral Detergent Fiber } \\
\hline Intake (g) & 1442.35 & 1496.58 & 1445.46 & 1417.14 & 187.89 & 0.955 & 0.797 \\
\hline Outgo (g) & 557.41 & 576.39 & 526.62 & 510.77 & 69.90 & 0.561 & 0.810 \\
\hline Digestibility (\%) & 61.34 & 61.73 & 63.53 & 63.91 & 0.465 & 0.002 & 0.992 \\
\hline \multicolumn{8}{|c|}{ Acid Detergent Fiber } \\
\hline Intake (g) & 861.59 & 892.68 & 855.86 & 829.04 & 111.29 & 0.855 & 0.782 \\
\hline Outgo (g) & 447.78 & 445.79 & 414.75 & 402.81 & 52.09 & 0.496 & 0.926 \\
\hline Digestibility (\%) & 48.02 & 50.07 & 51.59 & 51.42 & 0.214 & 0.000 & 0.001 \\
\hline \multicolumn{8}{|l|}{ Hemi cellulose } \\
\hline Intake (g) & 573.58 & 603.89 & 589.51 & 588.10 & 76.59 & 0.900 & 0.820 \\
\hline Outgo (g) & 111.53 & 122.42 & 111.87 & 107.96 & 15.32 & 0.764 & 0.642 \\
\hline Digestibility (\%) & 80.56 & 79.99 & 80.85 & 81.51 & 0.767 & 0.310 & 0.446 \\
\hline \multicolumn{8}{|l|}{ Cellulose } \\
\hline Intake (g) & 758.12 & 750.99 & 721.71 & 693.67 & 88.45 & 0.667 & 0.879 \\
\hline Outgo (g) & 313.47 & 300.11 & 278.83 & 269.05 & 32.49 & 0.319 & 0.958 \\
\hline Digestibility (\%) & 58.66 & 60.00 & 61.35 & 61.24 & 0.340 & 0.000 & 0.066 \\
\hline
\end{tabular}


From the table it has been revealed that feed intake per unit of gain was highest in control $\left(\mathrm{TD}_{0}\right)$ group followed by $\mathrm{TD}_{10}, \mathrm{TD}_{15}$ and $\mathrm{TD}_{5}$ i.e. the performance was better in group receiving $5 \%$ dried rice cake in ration and the poor result was seen in control group. The groups receiving $10 \%$ and $15 \%$ dried rice cake in experimental diets showed almost similar result. The results as fortnightly or as a whole was not statistically significant ( $P>0.05)$, though in all the groups showed numerically variable results.

Larson et al.,(1993) used wet distillers byproducts@0, 5.2, 12.6 and 40\% of DM in finishing ruminant and showed no significant $(\mathrm{P}=0.61)$ effect on gain: feed at $45^{\text {th }}$ day but significant $(\mathrm{P}=0.01)$ effect at the end of $(105$ days) trial. Ham et al., (1994) reported no significant effect $(\mathrm{P}>0.05)$ on gain: feed on finishing cattle using low, medium and high quality DDGS. Lodge et al., (1997) used wet and dry sorghum distiller's grains plus soluble in finishing cattle and found better feed: gain in dry distillers grains than that of wet distiller's grain. Similar observation was also reported by Al-Suwaiegh et al., (2002) where they used wet corn and sorghum distiller's grains and soluble resulted improved gain: feed efficiency in yearling finishing steer.

\section{Nutrient utilization through digestibility} trial by feeding dried rice cake at graded level in growing calves

A digestibility trial was conducted in growing calves by feeding graded level of dried rice cake to evaluate the nutrient utilization. The digestibility of nutrients namely dry matter (DM), crude protein (CP), crude fiber $(\mathrm{CF})$, ether extract (EE), nitrogen free extract (NEF), acid detergent fiber (ADF), neutral detergent fiber (NDF), cellulose and hemicellulose was present in Table 8. From the table it was found that $\mathrm{DM}, \mathrm{CP}, \mathrm{CF}$, and NFE digestibility were differed significantly
$(\mathrm{P}<0.05)$ on linear basis but there was no linear $(\mathrm{P}=0.086)$ or quadratic $(\mathrm{P}=0.132)$ effect on $\mathrm{EE}$ digestibility. Again NDF, ADF and cellulose digestibility were varied significantly $(\mathrm{P}<0.05)$ by dietary treatment of dried rice cake but there was no significant $(\mathrm{P}>0.05)$ change in hemi cellulose digestibility. The digestibility of nutrients in treatment groups always towards the higher side. The increased CP, CF, NFE, ADF, NDF and cellulose utilization in sun dried rice cake supplemented groups may be due to presence of higher concentration of live yeast (Saccharomyces cerevisiae) which alters the rumen fermentation (Carro et al., 1992).

The present findings corroborated with the findings of Peter et al., (2000), Birkelo et al., (2004) and Morghany et al., (2005) who showed positive effect of ADF, NDF, cellulose and hemicellose and CF digestibility by feeding wet corn distillers grain or live yeast supplementation. Again, Harrison et al., (1988) reported no significant improvement in apparent nutrient digestibility by feeding yeast culture in lactating cows. The similar observation was also reported by Hannah et al., (1990), Arambel et al., (1990) and Ham et al., (1994).Again the contrary observation also reported by Lodge et al., (1997) who observed lower organic matter, true and apparent nitrogen digestibility in lambs by feeding wet sorghum or corn distillers grain in lamb.

Feed intake was not affected by dietary supplementation of DRDGS. The final average body weights in different experimental groups were numerically better but statistically not significant. The same trend was also noticed in case of ADG. Effect on feed: gain either fortnightly or cumulative was not significant among various experimental groups. The better digestibility of organic nutrients was found in DRDGS treatment groups. 


\section{Acknowledgements}

Appreciation is extended to ICAR for providing all financial and laboratory support from AICRP on "Improvement of Feed Resources and Nutrient Utilization in Raising Animal Production" WBUAFS, Kolkata and Mr. Md. Chand for supplying male calf from Ganganagar Dairy Farm, Dum Dum.

\section{References}

Al-Suwaiegh, S., Fanning, K.C., Grant, R. J., Milton, C. T. and T.J. Klopfenstein. (2002) Utilization of distillers grains from the fermentation of sorghum or corn in diets for finishing beef and lactating dairy cattle. J. Anim. Sci. 80:1105-1111.

AOAC (1995). Official methods of analysis, Association of Official Analytical Chemist. Vol-I, 16 ${ }^{\text {th }}$ Ed., AOAC, International, Arlington, USA.

Arambel, M.J. and Kent B.A (1990). Effect of yeast culture on nutrient digestibility and milk yield response in early to midlactation. J. Dairy Sci. 73(6): 15601563.

Arambel, M.J. and Kent B.A (1990). Effect of yeast culture on nutrient digestibility and milk yield response in early to midlactation. J. Dairy Sci. 73(6): 15601563.

Bhat, P.N. and Taneja, V.K. (1998). Sustainable animal production systems in India: issues and approaches. Indian Journal of Animal Sciences, 68: 701712.

Birkelo, C.P., Brouk, M.J. and Schingoethe, D.J. (2004). The energy content of wet corn distillers grains for lactating dairy cows. J. Dairy Sci. 87:1815-1819.

Carro, M.D., Lebzien, P., and Rohr, K (1992). Effects of yeast culture on rumen fermentation, digestibility and duodenal flow in dairy cows fed silage based diet. Livest. Prod. Sci., 32:219-229.

Creighton, K.W., Wilson C.B., Klopfenstein, T.J. and Adams, D.C. (2003). Undegradable intake protein supplementation of compensating spring- born steers and summer-born steers during summer grazing. J. Anim. Sci., 81:791-799.

Ham, G.A., Stock, R.A, Klopfenstein, T.J., Larson, E.M, Stain D.H and Huffman R.P (1994).Wet distillers' byproducts compared with dried corn distillers grains with soluble as a source of protein and energy for ruminants. J.Anim.Sci. 72:3246-3257.

Hannah, S.M., Paterson, J.A., Williams, J.E. and Kerley, M.S. (1990). Effects of corn Vs Corn gluten feed on site, extent and ruminants of forage digestion and on rate and efficiency of gain. J. Anim. Sci. 68:2536-2545.

Harrison, G.A., Hemken, R.W., Dawson, K.A., Harmon, R.J. and Baker, K.B (1988). Influence of addition of yeast culture supplement to diets of lactating cows on ruminal fermentation and microbial population. J. Dairy Sci. 71:2967-2975.

Klopfenstein, T.J. (1996). Need for escape protein by grazing cattle. Anim. Feed Sci. Technol. 60:191-199.

Larson, E.M., Stock, R.A., Klopfenstein, T. J., Sindt, M.H. and Huffman, R.P. (1993). Feeding value of wet distillers' byproducts for finishing ruminants. $J$. Anim. Sci. 71:2228-2236.

Lodge, S.L., Stock, R.A., Klopfenstein, T.J., Shain, D.H. and Herold, D.W. (1997) Evaluation of wet distillers composite for finishing ruminants. J. Anim. Sci. 75:44-50.

Loy, T. W., Klopfenstein, T.J., Erickson, G.E., Macken, C.N. and MacDonald, J.C.(2003) Supplemental type and frequency on intake, performance and 
energy value of dry distillers grains in a high forage diet. J. Anim. Sci. 81(Suppl.2):107(Abstr.).

Martin J.L., Cupp, A.S., Rasby, R.J., Hall, Z.C. and Funston, R.N (2007). Utilization of dried distillers grains for developing beef heifers. J.Anim. Sci. 85:2298-2303.

Morghany, M., Sarhan, M.A., Abd El-Hey, A. and El-Tahan, A.A. (2005). Performance of lactating buffaloes fed rations supplemented with different levels of baker's yeast (Saccharomyces cerevisiae). Egyptian, J. Nutrition and Feeds (Special Issue) 8:21.

Morris. S., Klopfenstein, $\mathrm{T}$ and Adams D. (2005). Effect of dried distillers grains on heifer consumption of low or high quality forage. J. Anim. Sci. Vol 83(Suppl.2) p 82.

Nikerson, W.J., and Brawn, R.J. (1965).Uses and products of yeasts and yeast like fungi. Adv.Appl. Microbial., 7:225.
NRC (2001). Nutrient requirements of Dairy Cattle. $7^{\text {th }}$ ed. Natl. Acad. Press, Washington.

Peter, C.M., Faulkner, D.B., Merchen, N.R., Parrett, D.F., Nash, T.G. and Palmiquist, J.M. (2000). The effects of corn milling co-products growth performance and diet digestibility by beef cattle. J. Anim. Sci. 78:1-6.

Rosales, F.H. (1984). Yeast as protein source for human nutrition. Acta Microbiological Hungarica., 31:159.

SPSS Base Application Guide 10.0 (2000). SPSS. USA.

Van Soest, P.J., Robertson, J.B. and Lewis, B.A. (1991). Methods for dietary fiber, neutral detergent fiber and non-starch polysaccharides in relation to animal nutrition. J. Dairy Sci., 74:3583-3597.

\section{How to cite this article:}

Pakhira, M.C., P. Biswas and Mondal, M. 2018. Utilization of Dried Rice Distiller's Grains and Solubles (Rice Cake) on Growth Performance of Growing Male Calves. Int.J.Curr.Microbiol.App.Sci. 7(09): 1834-1844. doi: https://doi.org/10.20546/ijcmas.2018.709.223 